\title{
Awareness, Belief, and Communication Reaching Consensus
}

\author{
To Professor Masami Ito on the occasion of his 60's birthday \\ EMIKO FUKUDA $†$ emiko@valdes.titech.ac.jp \\ Graduate School of Decision Science and Technology, Tokyo Institute of Technology, \\ JAPAN \\ TAKASHI MATSUHISA* \\ mathisa@ge.ibaraki-ct.ac.jp \\ Department of Liberal Arts and Sciences, Ibaraki National College of Technology, JAPAN \\ HISATO SASANUMA ** \\ Undergraduate Course of Electric Engineering, Ibaraki National College of Technology, \\ Japan
}

\begin{abstract}
This article studies graph-theoretical conditions under which communication will lead to consensus among players about their decisions in circumstances where there are more than two players and they interact in pair without public announcement. It is shown that consensus on their decisions can be guaranteed if the communication graph contains no cycle. Where none of the requirements for player's knowledge is imposed as in the standard model of knowledge with partitional information structure.
\end{abstract}

Keywords: Awareness, belief, communication, consensus, agreeing to disagree.

\section{Introduction}

This article studies graph-theoretical conditions of communication under which players will reach consensus about their decisions in circumstances where there are more than two players and they interact in pairs without public announcement.

Recently researchers in such fields as economics, OR, and theoretical computer sciences have become interested in reasoning of belief and knowledge. There are pragmatic concerns about the relationship between knowledge (belief) and actions. Of most interest to us is the emphasis on situations

\footnotetext{
$\dagger$ Requests for reprints should be sent to Emiko Fukuda, Department of Value and Decision Science, Graduate School of Decision Science and Technology, Tokyo Institute of Technology, Oh-Okayama 2-Choume 12-1, Meguro-ku, Tokyo 152-8552, JAPAN.

* Supported by the Grants-in-Aid for Scientific Research(C)(2)(No.12640145 and No.12640145) in the Japan Society for the Promotion of Sciences.

** Current address: NHK (Nippon Houso Kyokai), Shibuya-ku, Tokyo 150-8081, Japan.
} 
involving the knowledge (belief) of a group of players rather than that of a single player. At the heart of any analysis of such situations as a conversation, a bargaining session or a protocol run by processes is the interaction between players. A player in a group must take into account not only events that have occurred in the world but also the knowledge of the other players in the group.

In some cases we need to consider the situation that the players has common-knowledge of an event; that is, simultaneously everyone knows the event, everyone knows that everyone knows the event, and so on. This notion also turns out to be a prerequisite for achieving agreement: In fact, Aumann [1] showed the famous agreement theorem; that is, if all posteriors of an event are common-knowledge among the players then the posteriors must be the same, even when they have different private information. This is precisely what makes it a crucial notion in the analysis of an interacting group of players.

Because the notion is defined by the infinite regress of all players' knowledge as above, common-knowledge is actually so unfeasible a tool in helping us analyze complicated situations involving groups of players. Thus we would like to remove it from our modelling.

In this regard, Geanakoplos and Polemarchakis [5] investigated a communication process in which two players announce their posteriors to each other. In the process players learn and revise their posteriors and they reach consensus without common-knowledge of an event. Furthermore, Krasucki [6] introduced the revision process of the players' information structures according to a communication graph, in which values of players' posteriors are communicated privately through messages among at least three players. He showed that in the process, consensus on the posteriors can be guaranteed if the communication graph contains no cycle. The result is an extension of the agreement theorem of Aumann [1].

All of the information structures in the models of Aumann [1], of Geanakoplos and Polemarchakis [5] and of Krasucki [6] are given by information partitions on a state space. Bacharach [2] showed that the information partition model is equivalent to his knowledge operator model with the three axioms about the operators: $\mathbf{T}$ axiom of knowledge (what is known is true), $\mathbf{4}$ axiom of transparency (that we know what we do know) and $\mathbf{5}$ axiom of wisdom (that we know what we do not know.) He pointed out that the assumptions for the partition are problematic in decision making, and hence the model of analyzing complicated situations should be also constructed without such strong assumptions.

Matsuhisa and Kamiyama [7] introduced the lattice structure of knowledge for which the requirements such as the three axioms are not imposed, 
and they succeeded in extending Aumann's theorem to their model. However the knowledge operators satisfy the monotonicity property, which implies that the players have logically omniscient ability; i.e., $i$ knows (believes) all logical consequences of what $i$ knows (believes).

Matsuhisa and Usami [9] presented an awareness operator model in which the operators are not required to satisfy the monotonicity property. They extended Aumann's theorem to the awareness model. Both of the agreement theorems are however established under the common-knowledge (or common-belief) assumption.

The purpose of this article is to introduce a communication process in the awareness operator model of Matsuhisa and Usami [9], and to extend the agreement theorem to the model without common-belief assumption in the line of Krasucki [6]. Our result is:

Main theorem. In the communication process associated with an awareness operator model, consensus can be guaranteed if the communication protocol is an acyclic graph.

This is an extension of the result in Matsuhisa, Tominaga and Kamiyama [8] where they showed the similar result in the lattice structure of knowledge.

This article is organized as follows: Section 2 presents the three notions: an awareness belief model, the associated information structure and the communication process. Further we introduce the notion of consensus of the values of a decision function. In Section 3 we illustrate Main theorem by using a simple example. Section 4 gives the proof of the theorem, and we will end by concluding remarks.

\section{The Model}

Let $\Omega$ be a non-empty finite set called a state space, $N$ a set of finitely many at least two players, and let $\mathcal{F}$ denote the field $2^{\Omega}$ that is the family of all subsets of $\Omega$. Each member of $\mathcal{F}$ is called an event and each element of $\Omega$ called a state.

\subsection{Belief and common-belief}

$A$ belief structure is a tuple $\left\langle\Omega,\left(B_{i}\right)_{i \in N}\right\rangle$ in which $B_{i}: \mathcal{F} \rightarrow \mathcal{F}$ is $i$ 's belief operator. The interpretation of the event $B_{i} F$ is that ' $i$ believes $F$.' The mutual belief operator $B_{E} F$ on $2^{\Omega}$ is defined by

$$
B_{E} F=\bigcap_{i \in N} B_{i} F,
$$


which is interpreted as that 'everybody believes $F$.' A common-belief operator is an operator $B_{C}: \mathcal{F} \rightarrow \mathcal{F}$ satisfying the fixed point property:

$$
B_{C} F \subseteq B_{E}\left(F \cap B_{C} F\right) \quad \text { for every } F \text { of } \mathcal{F} .
$$

We say that an event $E$ is common-belief at $\omega$ if $\omega$ belongs to $B_{C} E$.

Example 1: We shall give formally the iterated notion of commonknowledge mentioned in Introduction: Let $\left\{C^{m}\right\}_{m=0}^{\infty}$ be the descending chain of operators on $\mathcal{F}$ defined by

$$
\begin{aligned}
C^{0} F & :=B_{E} F ; \quad \bar{C}^{m-1} F:=B_{E}\left(F \cap C^{m-1} F\right) ; \\
C^{m} F & :=\bar{C}^{m-1} F \cap C^{m-1} F .
\end{aligned}
$$

The iterated common-knowledge operator $C$ is the infimum of the chain:

$$
C F=\bigcap_{m=0,1,2, \cdots} C^{m} F .
$$

It is plainly observed that $C$ satisfies Axiom $\mathbf{F P}$, and that $C F$ is interpreted as simultaneously everyone knows $F$, everyone knows that everyone knows $F$, and so on.

\subsection{Awareness and belief}

We present a model of awareness according to Matsuhisa and Usami [9]. This model follows from E. Dekel, B. L. Lipman and A. Rustichini [3]. A different approach of awareness models is discussed in R. Fagin, J.Y. Halpern, Y. Moses and M.Y. Vardi [4] .

An awareness structure is a tuple $\left\langle\Omega,\left(A_{i}\right)_{i \in N},\left(B_{i}\right)_{i \in N}\right\rangle$ in which $\left\langle\Omega,\left(B_{i}\right)_{i \in N}\right\rangle$ is a belief structure and $A_{i}$ is $i$ 's awareness operators on $\mathcal{F}$ such that Axiom PL (axiom of plausibility) is valid:

PL $\quad B_{i} F \cup B_{i}\left(\Omega \backslash B_{i} F\right) \subseteq A_{i} F \quad$ for every $F$ in $\mathcal{F}$.

The interpretation of $A_{i} F$ is that ' $i$ is aware of $F$.' The axiom $\mathbf{P L}$ says that $i$ is aware of $F$ if he believes it or if he believes that he does not believe it. ${ }^{1}$ The mutual awareness operator $A_{E}$ on $\mathcal{F}$ is defined by

$$
A_{E} F=\bigcap_{i \in N} A_{i} F,
$$


where $A_{E} F$ is interpreted as the event 'everybody is aware of $F$.'

We say that an event $F$ is self-aware of $i$ if $F \subseteq A_{i} F$ and it is said to be publicly aware if $F \subseteq A_{E} F$. An event $T$ is said to be i's evident belief if $T \subseteq B_{i} T$, and it is said to be public belief at state $\omega$ if $\omega \in T \subseteq B_{E} T$. An event is public belief (or respectively, it is publicly aware) if whenever it occurs all players believe it (or they are all aware of it.) We can think of public belief as embodying the essence of what is involved in all players making their direct observations. An awareness structure is called finite if the state-space is finite.

\subsection{Associated information structure}

M. Bacharach [2] introduces the strong epistemic model that coincides with the Kripke semantics corresponding to the modal logic $\mathbf{S 5} .^{2}$

Further he defines the information partition induced from the knowledge operator of the epistemic model. ${ }^{3}$ Following his line we generalize the notion of information partition as follows.

The associated information structure $\left(P_{i}\right)_{i \in N}$ with an awareness structure $\left\langle\Omega,\left(A_{i}\right),\left(B_{i}\right)\right\rangle$ is the class of mappings $P_{i}$ of $\Omega$ into $\mathcal{F}$ defined by

$$
P_{i}(\omega)=\bigcap_{T \in 2^{\Omega}}\left\{T \mid \omega \in T \subseteq B_{i} T\right\} .
$$

(If there is no event $T$ for which $\omega \in T \subseteq B_{i} T$ then we take $P_{i}(\omega)$ to be non-defined.) We call $P_{i}(\omega)$ the $i$ 's evidence set at $\omega$, which is interpreted as the basis for all $i$ 's evident beliefs. This is because each $i$ 's evident belief $T$ is decomposed into a union of all evidence sets contained in $T$.

Remark. The strong epistemic model can be interpreted as the awareness structure $\left\langle\Omega,\left(A_{i}\right),\left(B_{i}\right)\right\rangle$ such that $B_{i}$ satisfies $\mathbf{N}, \mathbf{K}, \mathbf{T}, \mathbf{4}$ and $\mathbf{5}$. In this situation it is easily verified that any awareness operators $A_{i}$ must be the trivial operator; i.e. $A_{i}(E)=\Omega$ for every $E \in \mathcal{F}$. This says that an awareness structure is an extension of the strong epistemic model.

\subsection{Posterior revised}

Let $\mu$ be a probability measure on $\mathcal{F}$. By $i$ 's posterior of an event $X$ we usually mean the conditional probability $\mu\left(X \mid P_{i}(\omega)\right)$. We improve on this notion as follows: ${ }^{4}$ Let $\left\langle\Omega,\left(A_{i}\right),\left(B_{i}\right), \mu\right\rangle$ be an awareness structure with a common-prior $\mu$ and $X$ an event. For every positive real number $q_{i}$, we 
denote

$$
\left[q_{i}\right]=\left\{\omega \in \Omega \mid \mu\left(X \cap A_{i}(X) \mid P_{i}(\omega)\right)=q_{i}\right\} .
$$

The interpretation of $\mu\left(X \cap A_{i}(X) \mid P_{i}(\omega)\right)$ is the conditional probability of the $i$ 's awareness section of $X$ under his evidence set at $\omega$, and $\left[q_{i}\right]$ is interpreted as the event that $i$ 's posterior is $q_{i}$. We say $q_{i}$ to be $i$ 's posterior of $X$ at $\omega$ if $\omega$ belongs to $\left[q_{i}\right]$. We denote by $q$ the profile $\left(q_{i}\right)_{i \in N}$. The event $[q]$ denotes the intersection

$$
[q]=\bigcap_{i \in N}\left[q_{i}\right]
$$

We say that the players commonly believe their posteriors $q_{i}$ of $X$ at $\omega$ if $\omega \in B_{C}([q])$.

In this circumstances we can obtain the generalized version of the agreement theorem of Aumann:

Proposition 1 Let $X$ be a self-aware event. Suppose all players have the common-prior and $q_{i}$ is $i$ 's posterior of $X$ at $\omega$. If $\omega \in B_{C}([q])$ then $q_{i}=q_{j}$ for any $i, j$.

Proof: Will be given by the same line of Theorem B in Matsuhisa and Usami [9]. We shall give it in Appendix B for readers' convenience.

\subsection{Decision function ${ }^{5}$}

Let $Z$ be a set of decisions for all players in $N$. An $i$ 's decision function is a mapping $f_{i}$ of $\mathcal{F}$ into $Z$. It is said to satisfy the sure thing principle if it is preserved under disjoint union; that is, for every pair of disjoint events $S$ and $T$ such that if $f_{i}(S)=f_{i}(T)=d$ then $f_{i}(S \cup T)=d$.

A decision function $f_{i}$ is said to be preserved under difference if for all events $S$ and $T$ such that $S \subseteq T, f_{i}(S)=f_{i}(T)=d$ then we have $f_{i}(T \backslash S)=d$.

If $f_{i}$ is intended to be a posterior probability, we assume given a probability measure $\mu$ which is common for all players and some event $X$. Then $f_{i}$ is the mapping of the domain of $\mu$ into the closed interval $[0,1]$ such that

$$
f_{i}(E)=\mu\left(X \cap A_{i}(X) \mid E\right),
$$

where $\mu(E) \neq 0$. We plainly observe that this $f_{i}$ satisfies the sure thing principle and is preserved under difference. 


\subsection{Communication with awareness structure}

We assume that players communicate by sending messages. A protocol is a mapping $\operatorname{Pr}$ of the set of non-negative integers $\mathbf{Z}_{+}$into the product set $N \times N$ that assigns to each $t$ a pair of players $(s(t), r(t))$. Here $t$ stands for time and $s(t)$ and $r(t)$ are, respectively, the sender and the recipient of the communication which takes place at time $t$. We consider a protocol as the directed graph whose vertices are the set of all players $N$ and such that there is an edge (or an arc) from $i$ to $j$ if and only if there are infinitely many $t$ such that $s(t)=i$ and $r(t)=j$.

A protocol is said to be fair if the graph is strongly-connected; in words, every player in this protocol communicates directly or indirectly with every other player infinitely often. It is said to contain a cycle if the graph contains a cyclic path; that is, there are players $i_{1}, i_{2}, \ldots, i_{k}$ with $k \geq 3$ such that for all $m<k, i_{m}$ communicates directly with $i_{m+1}$, and such that $i_{k}$ communicates directly with $i_{1}$.

Definition. A communication process $\pi$ of revisions of the values of decision functions $\left(f_{i}\right)_{i \in N}$ is a triple $\left\langle\operatorname{Pr},\left(Q_{i}^{t}\right)_{(i, t) \in N \times \mathbf{Z}_{+}},\left(f_{i}\right)_{i \in N}\right\rangle$, in which $\operatorname{Pr}(t)=(s(t), r(t))$ is a fair protocol such that for every $t, r(t)=s(t+1)$, communications proceed in rounds $s^{6}$, and $Q_{i}^{t}$ is the mapping of $\Omega$ into $\mathcal{F}$ for $i$ at time $t$ that is defined inductively as follows:

- We assume given a mapping $Q_{i}^{0}:=P_{i}$.

- Suppose $Q_{i}^{t}$ is defined.

- $d_{i}^{t}(\omega)$ denotes the value $f_{i}\left(Q_{i}^{t}(\omega)\right)$ :

- $W_{i}^{t}$ is the mapping of $\Omega$ into $\mathcal{F}$ that assigns to each state $\omega$ the event $W_{i}^{t}(\omega)$ consisting of all the states $\xi$ such that $d_{i}^{t}(\xi)=d_{i}^{t}(\omega)$ :

- $B_{i}^{t}$ is the belief operator induced by $Q_{i}^{t}$ defined by $B_{i}^{t} E=\{\omega \in$ $\left.\Omega \mid Q_{i}^{t}(\omega) \subseteq E\right\}:$

- The recipient $j=r(t)$ at $t$ sends the message $B_{j}^{t}\left(W_{j}^{t}(\omega)\right)$ at $t+1$ when $\omega \in B_{j}^{t}\left(W_{j}^{t}(\omega)\right)$, and he does not send the message otherwise.

- Then $Q_{i}^{t+1}(\omega)$ is defined as follows:

- If $i$ is not a recipient of a message at time $t+1$, then $Q_{i}^{t+1}(\omega)=$ $Q_{i}^{t}(\omega)$.

- If $i$ is a recipient of a message at time $t+1$, then $Q_{i}^{t+1}(\omega)=$ $Q_{i}^{t}(\omega) \cap B_{s(t)}^{t}\left(W_{s(t)}^{t}(\omega)\right)$. 
Specifically the sender $j$ sends to $i$ the message that he believes that his decision is $d_{j}^{t}(\omega)$. The communication is said to be cut off in a state $\omega$ if $\omega \notin B_{j}^{t}\left(W_{j}^{t}(\omega)\right)$ for some $t$ and the recipient $j=r(t)$ at $t$.

\subsection{Consensus}

The family $\left\{Q_{i}^{t}(\omega) \mid t=0,1,2, \ldots\right\}$ is a descending chain in $\mathcal{F}$ and so the limit $Q_{i}^{\infty}$ exists in each state where the communication is never cut off: In fact, there exists sufficiently large $T \in \mathbf{Z}_{+}$such that for all $t \geq T, Q_{i}^{t}=Q_{i}^{T}$ because $\Omega$ is finite. Since the protocol is fair, there exist infinitely many $t$ such that $r(t)=i$, it follows that $Q_{i}^{\infty}(\omega)=\lim _{t \rightarrow \infty} Q_{i}^{t}(\omega)$.

Remark. For each $i$ the sequence of the domains of $Q_{i}^{t}(t=0,1, \cdots)$ makes a descending chain, and thus it may be that $Q_{i}^{\infty}(\omega)=\emptyset$ at some $\omega$.

In view of the above discussion it can be easily observed that

Lemma 1 For every player $i$, the sequence $\left\{d_{i}^{t}(\omega) \mid t=0,1,2, \ldots\right\}$ converges to the limiting value $d_{i}^{\infty}(\omega)=\lim _{t \rightarrow \infty} d_{i}^{t}(\omega)$ of $f$ at each state $\omega$ if at $\omega$ the communication is never cut off and $Q_{i}^{\infty}(\omega)$ is defined.

We call $d_{i}^{\infty}(\omega)=f\left(Q_{i}^{\infty}(\omega)\right)$ the limiting value of $f$ at $\omega$ for $i$.

Definition. We say that consensus on the limiting values of decision functions $\left(f_{i}\right)_{i \in N}$ can be guaranteed in the communication process if $d_{i}^{\infty}(\omega)$ and $d_{j}^{\infty}(\omega)$ are equal for each player $i, j$ and in all the states $\omega$ that the communication is never cut off and that $Q_{i}^{\infty}$ is defined for all $i \in N$.

Remark. In the communication process, the limiting value of $f$ can be reached in finitely many rounds; i.e., there exists a non-negative integer $T$ independent of $\omega$ such that for every player $i$ and for all $t \geq T, d_{i}^{\infty}(\omega)=$ $d_{i}^{t}(\omega)$ by Lemma 1.

\section{Example}

We show a simple example below in order to illustrate Main theorem in Section 1. We consider the awareness structure $\left\langle\Omega,\left(A_{i}\right)_{i=A, B, C},\left(B_{i}\right)_{i=A, B, C}\right\rangle$ among three players $A, B$ and $C$ as follows: $\Omega$ is the eight point set $\left\{\omega_{1}, \omega_{2}, \ldots, \omega_{8}\right\}$ as state space, the mapping $Q_{i}^{0}: \Omega \rightarrow \mathcal{F}$ is defined by Figure 1: Say

$$
\begin{aligned}
& Q_{A}^{0}\left(\omega_{1}\right)=Q_{A}^{0}\left(\omega_{2}\right)=Q_{A}^{0}\left(\omega_{3}\right)=Q_{A}^{0}\left(\omega_{4}\right)=\left\{\omega_{1}, \omega_{2}, \omega_{3}, \omega_{4}\right\} \\
& Q_{A}^{0}\left(\omega_{5}\right)=Q_{A}^{0}\left(\omega_{7}\right)=\left\{\omega_{5}, \omega_{6}, \omega_{7}, \omega_{8}\right\} \\
& Q_{A}^{0}\left(\omega_{6}\right)=Q_{A}^{0}\left(\omega_{8}\right)=\left\{\omega_{6}, \omega_{8}\right\} .
\end{aligned}
$$


Table 1. The message will be sent by $A$ at $t=1$.

\begin{tabular}{|c||c|c|c|c|c|c|c|c|}
\hline$t=1$ & $\omega_{1}$ & $\omega_{2}$ & $\omega_{3}$ & $\omega_{4}$ & $\omega_{5}$ & $\omega_{6}$ & $\omega_{7}$ & $\omega_{8}$ \\
\hline$d_{A}^{0}$ & $\frac{1}{2}$ & $\frac{1}{2}$ & $\frac{1}{2}$ & $\frac{1}{2}$ & $\frac{1}{2}$ & 0 & $\frac{1}{2}$ & 0 \\
\hline$W_{A}^{0}$ & 123457 & 123457 & 123457 & 123457 & 123457 & 68 & 123457 & 68 \\
\hline$B_{A}^{0}\left(W_{A}^{0}\right)$ & 1234 & 1234 & 1234 & 1234 & 1234 & 68 & 1234 & 68 \\
\hline
\end{tabular}

$i$ 's belief operator $B_{i}$ is defined by $B_{i}(E)=\left\{\omega \in \Omega \mid Q_{i}^{0}(\omega) \subseteq E\right\}$ for $i=A, B, C$, and $A_{i}$ is the trivial operator on $\mathcal{F}$. Then it is easily observed that the class of mappings $\left\{Q_{i}^{0}\right\}_{i \in\{A, B, C}$ coincides with the associated information structures at time $t=0$ with the awareness structure $\left\langle\Omega,\left(A_{i}\right)_{i \in\{A, B, C\}},\left(B_{i}\right)_{i \in\{A, B, C\}}\right\rangle$.

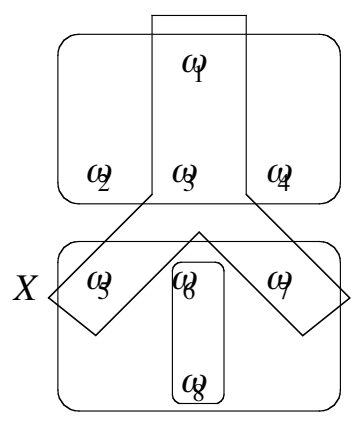

Q

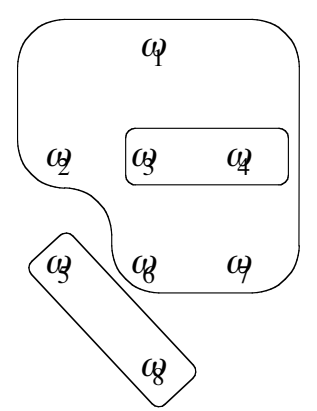

$Q_{B}$

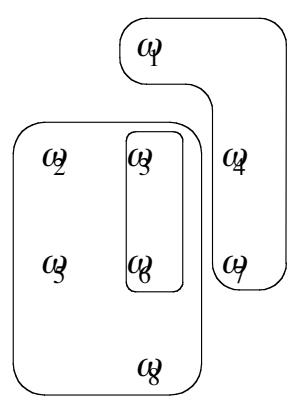

$Q$

Figure 1. Initial information structure.

We let $X$ be the event $\{1,3,5,7\}^{7}$ and consider the protocol $A \rightleftharpoons B \rightleftharpoons C$ that contains no cycle. Let us give the common prior $\mu(\omega)=\frac{1}{8}$ for each state $\omega \in \Omega$, and the common decision function $f$ defined by $f(E)=$ $\mu(X \mid E)$. It is noted that $f$ satisfies the sure thing principle and is preserved under difference. Then we obtain the message $B_{A}^{0}\left(W_{A}^{0}\right)$ of $A$ at $t=0$ given in Table 1.

Player $A$ sends the message $B_{A}^{0}\left(W_{A}^{0}(\omega)\right)$ to the recipient $B$, and he revises his information structure as follows: 
Table 2. The message will be sent by $B$ at $t=2$.

\begin{tabular}{|c||c|c|c|c|c|c|c|c|}
\hline$t=2$ & $\omega_{1}$ & $\omega_{2}$ & $\omega_{3}$ & $\omega_{4}$ & $\omega_{5}$ & $\omega_{6}$ & $\omega_{7}$ & $\omega_{8}$ \\
\hline$d_{B}^{1}$ & $\frac{1}{2}$ & $\frac{1}{2}$ & $\frac{1}{2}$ & $\frac{1}{2}$ & $\times$ & 0 & $\times$ & 0 \\
\hline$W_{B}^{1}$ & 1234 & 1234 & 1234 & 1234 & $\times$ & 68 & $\times$ & 68 \\
\hline$B_{B}^{1}\left(W_{B}^{1}\right)$ & 1234 & 1234 & 1234 & 1234 & $\times$ & 68 & $\times$ & 68 \\
\hline
\end{tabular}

Table 3. The message will be sent by $C$ at $t=3$.

\begin{tabular}{|c||c|c|c|c|c|c|c|c|}
\hline$t=3$ & $\omega_{1}$ & $\omega_{2}$ & $\omega_{3}$ & $\omega_{4}$ & $\omega_{5}$ & $\omega_{6}$ & $\omega_{7}$ & $\omega_{8}$ \\
\hline$d_{C}^{2}$ & $\frac{1}{2}$ & $\frac{1}{2}$ & 1 & $\frac{1}{2}$ & $\times$ & 0 & $\times$ & 0 \\
\hline$W_{C}^{2}$ & 124 & 124 & 3 & 124 & $\times$ & 68 & $\times$ & 68 \\
\hline$B_{C}^{2}\left(W_{C}^{2}\right)$ & 14 & 14 & 3 & 14 & $\times$ & 68 & $\times$ & 68 \\
\hline
\end{tabular}

$$
\begin{aligned}
& Q_{B}^{1}\left(\omega_{1}\right)=Q_{B}^{1}\left(\omega_{2}\right)=\left\{\omega_{1}, \omega_{2}, \omega_{3}, \omega_{4}\right\} \\
& Q_{B}^{1}\left(\omega_{3}\right)=Q_{B}^{1}\left(\omega_{4}\right)=\left\{\omega_{3}, \omega_{4}\right\}, \quad Q_{B}^{1}\left(\omega_{6}\right)=\left\{\omega_{6}\right\}, \quad Q_{B}^{1}\left(\omega_{8}\right)=\left\{\omega_{8}\right\}
\end{aligned}
$$

Note that $Q_{B}^{1}$ is not defined at each $\omega_{5}, \omega_{7}$. Next the message will be sent by $B$ at $t=2$ is given in Table 2 .

Then $C$ 's revised information structure at $t=2$ and the message sent by $C$ are as follows:

$$
\begin{aligned}
& Q_{C}^{2}\left(\omega_{1}\right)=Q_{C}^{2}\left(\omega_{4}\right)=\left\{\omega_{1}, \omega_{4}\right\} \quad Q_{C}^{2}\left(\omega_{2}\right)=\left\{\omega_{2}, \omega_{3}\right\}, \\
& Q_{C}^{2}\left(\omega_{3}\right)=\left\{\omega_{3}\right\}, \quad Q_{C}^{2}\left(\omega_{6}\right)=\left\{\omega_{6}\right\}, \quad Q_{C}^{2}\left(\omega_{8}\right)=\left\{\omega_{6}, \omega_{8}\right\} .
\end{aligned}
$$

The communication goes on and then $B$ 's revised information structure at $t=3$ is given by

$$
\begin{aligned}
& Q_{B}^{3}\left(\omega_{1}\right)=Q_{B}^{3}\left(\omega_{4}\right)=\left\{\omega_{1}, \omega_{4}\right\} \\
& Q_{B}^{3}\left(\omega_{3}\right)=\left\{\omega_{3}\right\}, \quad Q_{B}^{3}\left(\omega_{6}\right)=\left\{\omega_{6}\right\}, \quad Q_{B}^{3}\left(\omega_{8}\right)=\left\{\omega_{8}\right\},
\end{aligned}
$$

Player $A$ 's revised information structure at $t=4$ is given by

$$
\begin{aligned}
& Q_{A}^{4}\left(\omega_{1}\right)=Q_{A}^{4}\left(\omega_{4}\right)=\left\{\omega_{1}, \omega_{4}\right\} \\
& Q_{A}^{4}\left(\omega_{3}\right)=\left\{\omega_{3}\right\}, \quad Q_{A}^{2}\left(\omega_{6}\right)=Q_{A}^{2}\left(\omega_{8}\right)=\left\{\omega_{6}, \omega_{8}\right\},
\end{aligned}
$$

Player $B$ 's revised information structure at $t=5$ is given by as follows:

$$
\begin{aligned}
& Q_{B}^{5}\left(\omega_{1}\right)=Q_{B}^{5}\left(\omega_{4}\right)=\left\{\omega_{1}, \omega_{4}\right\} \\
& Q_{B}^{5}\left(\omega_{3}\right)=\left\{\omega_{3}\right\}, \quad Q_{B}^{5}\left(\omega_{6}\right)=\left\{\omega_{6}\right\}, \quad Q_{B}^{5}\left(\omega_{8}\right)=\left\{\omega_{8}\right\}
\end{aligned}
$$


Table 4. The message will be sent by $B$ at $t=4$.

\begin{tabular}{|c||c|c|c|c|c|c|c|c|}
\hline$t=4$ & $\omega_{1}$ & $\omega_{2}$ & $\omega_{3}$ & $\omega_{4}$ & $\omega_{5}$ & $\omega_{6}$ & $\omega_{7}$ & $\omega_{8}$ \\
\hline$d_{B}^{3}$ & $\frac{1}{2}$ & $\times$ & 1 & $\frac{1}{2}$ & $\times$ & 0 & $\times$ & 0 \\
\hline$W_{B}^{3}$ & 14 & $\times$ & 3 & 14 & $\times$ & 68 & $\times$ & 68 \\
\hline$B_{B}^{3}\left(W_{B}^{3}\right)$ & 14 & $\times$ & 3 & 14 & $\times$ & 68 & $\times$ & 68 \\
\hline
\end{tabular}

Table 5. The message will be sent by $A$ at $t=5$.

\begin{tabular}{|c||c|c|c|c|c|c|c|c|}
\hline$t=5$ & $\omega_{1}$ & $\omega_{2}$ & $\omega_{3}$ & $\omega_{4}$ & $\omega_{5}$ & $\omega_{6}$ & $\omega_{7}$ & $\omega_{8}$ \\
\hline$d_{A}^{4}$ & $\frac{1}{2}$ & $\times$ & 1 & $\frac{1}{2}$ & $\times$ & 0 & $\times$ & 0 \\
\hline$W_{A}^{4}$ & 14 & $\times$ & 3 & 14 & $\times$ & 68 & $\times$ & 68 \\
\hline$B_{A}^{4}\left(W_{A}^{4}\right)$ & 14 & $\times$ & 3 & 14 & $\times$ & 68 & $\times$ & 68 \\
\hline
\end{tabular}

The message will be sent by $B$ at $t=6$ is given by Table 6 .

Table 6 . The message will be sent by $B$ at $t=6$.

\begin{tabular}{|c||c|c|c|c|c|c|c|c|}
\hline$t=6$ & $\omega_{1}$ & $\omega_{2}$ & $\omega_{3}$ & $\omega_{4}$ & $\omega_{5}$ & $\omega_{6}$ & $\omega_{7}$ & $\omega_{8}$ \\
\hline$d_{B}^{5}$ & $\frac{1}{2}$ & $\times$ & 1 & $\frac{1}{2}$ & $\times$ & 0 & $\times$ & 0 \\
\hline$W_{B}^{5}$ & 14 & $\times$ & 3 & 14 & $\times$ & 68 & $\times$ & 68 \\
\hline$B_{B}^{5}\left(W_{B}^{5}\right)$ & 14 & $\times$ & 3 & 14 & $\times$ & 68 & $\times$ & 68 \\
\hline
\end{tabular}

It is observed that for each $i,\left\{Q_{i}^{t}(\omega) \mid t=0,1,2, \cdots\right\}$ becomes stationary and $Q_{i}^{t}(\omega)$ is given as in Figure 2. All values of decision functions are equal. Therefore consensus can be guaranteed in this example. (Note that $Q_{i}^{t}(\omega)$ is not defined at each state $\omega_{2}, \omega_{5}$ and $\omega_{7}$.)

\section{The Result}

\subsection{Main theorem}

We can now state our main theorem explicitly as below and we shall prove:

THEOREM 1 In the communication process associated with awareness structure, suppose that the all decision functions are common for all players, and 
that the common decision function satisfies the sure thing principle and is preserved under difference. Consensus on the limiting values of the decision function can be guaranteed if the protocol contains no cycle.

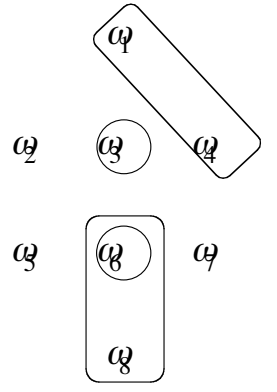

$q^{6}$

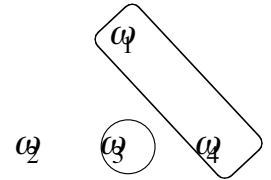

a) 19

(18)

$Q_{b}^{6}$

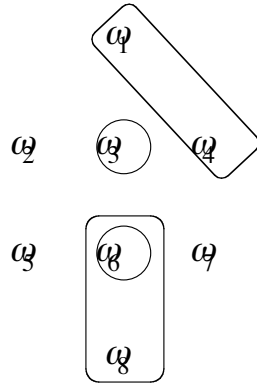

$Q^{6}$

Figure 2. Revised information structure at $t=6$.

The following corollary is Main theorem in Introduction; an extension of the agreement theorems of Geanakoplos and Polemarchakis [5] and Matsuhisa and Kamiyama [7]. This follows from Theorem 1 in considering that the common decision function is given by the revised posterior (Section 2.5.)

Corollary 1 Suppose that all players have a common-prior. Consensus on the limiting values of the posteriors for a publicly aware event among all players can still be guaranteed in the communication if the protocol contains no cycle.

\section{2. $\quad R T$-information structure}

Before proceeding to the proof, we need the notion of $R T$-information structure and the fundamental lemma:

A mapping $Q: \Omega \rightarrow \mathcal{F}$ is said to be an RT-information structure ${ }^{8}$ on $\Omega$ if the following two conditions are true: For each $i$ and for every state $\omega$ at which $Q(\omega)$ is defined,

Ref $\omega \in Q(\omega)$;

Trn $\quad \xi \in Q(\omega)$ implies $\quad Q(\xi) \subseteq Q(\omega)$. 
The following lemma is a key to proving the theorem:

Fundamental lemma. ${ }^{9}$ Let $\omega$ be a state in $\Omega$, and $Q$ an RT-information structure on $\Omega$. Suppose that $f$ be a decision function that satisfies the sure thing principle and is preserved under difference.

If there exists an event $H$ of $\mathcal{F}$ such that the two conditions are true: For each $\xi$ of $H$,

(a) $Q(\xi)$ is defined and it always contained in $H$, and

(b) it is always satisfied that $f(Q(\xi))=f(Q(\omega))$,

then we obtain that

$$
f(H)=f(Q(\omega)) .
$$

Proof: See Appendix A.

\subsection{Proof of theorem 1}

It is sufficient to prove the following theorem: In fact, on noting that the protocol has no cycle, Theorem 1 immediately follows from Theorem 2 by inductive steps on the players who are connected directly.

THEOREM 2 In a communication process associated with an awareness structure, suppose that the the decision functions are common for all players, and that the common decision function satisfies the sure thing principle and is preserved under difference. If player $i$ communicates his/her message directly to player $j$ then their limiting values of the decision function must be equal.

Proof: Let $\pi=\left\langle\operatorname{Pr},\left(Q_{i}^{t}\right), f\right\rangle$ be a communication process associated with awareness structures where $f$ is the common decision function. The protocol has the property that, if $s(t)=i$ and $r(t)=j$ then $r(t+m)=i$ and $s(t+m)=j$ for some $m$.

It should be noted that $P_{i}$ is an $R T$-information structure, and so is $Q_{i}^{\infty}$ for every $i$. Therefore in view of (Ref) and (Trn), it can be plainly observed that

$$
\begin{array}{ll}
\text { T } & B_{i}^{\infty}(F) \subseteq F \\
4 & B_{i}^{\infty}(F) \subseteq B_{i}^{\infty}\left(B_{i}^{\infty}(F)\right) .
\end{array}
$$


For each state $\omega$ at which $Q_{i}^{\infty}$ is defined, set $H=B_{i}^{\infty}\left(W_{i}^{\infty}(\omega)\right) \cap$ $B_{j}^{\infty}\left(W_{j}^{\infty}(\omega)\right)$ and $Q=Q_{l}^{\infty}$ for $l=i, j$. To complete the proof, it suffices to verify the two properties:

(a) If $\xi$ is a member of $H$ then $Q(\xi) \subseteq H$, and

(b) $H$ is contained in the set consisting of all the states $\xi$ such that $d_{l}^{\infty}(\xi)=$ $d_{l}^{\infty}(\omega)$.

Indeed, if it is the case then, viewing of Fundamental Lemma we obtain that $f(H)=f\left(Q_{l}^{\infty}(\omega)\right)$ for each $l \in\{i, j\}$, and thus $d_{i}^{\infty}(\omega)=d_{j}^{\infty}(\omega)$ as required.

Proof of (a) : It follows from 4 that $Q_{l}^{\infty}(\xi) \subseteq B_{l}^{\infty}\left(W_{l}^{\infty}(\omega)\right)$, and it follows from the definition of $Q_{l}^{t+1}$ that $\xi \in Q_{l}^{\infty}(\xi) \subseteq B_{l^{\prime}}^{\infty}\left(W_{l^{\prime}}^{\infty}(\xi)\right)$ for $l^{\prime} \in\{i, j\} \backslash\{l\}$, and thus $W_{l^{\prime}}^{\infty}(\xi)=W_{l^{\prime}}^{\infty}(\omega)$ by $\mathbf{T}$. We observe that for every $\xi \in H, Q(\xi) \subseteq B_{i}^{\infty}\left(W_{i}^{\infty}(\omega)\right) \cap B_{j}^{\infty}\left(W_{j}^{\infty}(\omega)\right)$.

Proof of (b): In view of $\mathbf{T}$ it is easily observed that $H \subseteq W_{l}^{\infty}(\omega)=\{\xi \in$ $\left.\Omega \mid d_{l}^{\infty}(\xi)=d_{l}^{\infty}(\omega)\right\}$, in completing the proof.

\section{Concluding Remarks}

Our real concern in this article is about relationship between players' beliefs and their decision making, especially when and how the players reach consensus about their decisions.

We focus on extending the agreeing theorem of Aumann [1] to an 'awareness and belief' model in the line of Krasucki [6]. We have shown that the nature that consensus can be guaranteed in a communication process is dependent not on common-belief assumption but on the updating with awareness and belief when each player receives information.

It can be seen that the notion of awareness does not play a role in the agreement theorem in the case of decision functions, but it does play an essential role in the probabilistic version of the theorem. In the analysis of decision theoretical situations, it seems to be problematic to keep the tension between allowing for awareness and assuming a common prior that needs to be sorted out at a more fundamental level, and thus it is more problematic to do so between allowing fo awareness and assuming common decision function. This issue of updating with awareness concerning with decision functions cannot be discussed at all in this article. There is here a research agenda of potential interest which we hope to pursue further. 


\section{Acknowledgments}

We want to thank the referee for his/her comments to improve this article.

\section{Appendix A}

\section{Proof of Fundamental Lemma}

We set by $\mathcal{S}$ the class of all the components $A(\xi)$ of $Q(\omega)$ such that $f(A(\xi))=f(Q(\omega))$. We divide two cases: First that $\mathcal{S} \neq \emptyset$, and secondly that $\mathcal{S}=\emptyset$.

Case I. $\mathcal{S} \neq \emptyset$ : Denote by $S$ the union of all members of $\mathcal{S}$ that is not empty. To prove the lemma in this case it suffices to show that $S=Q(\omega)$. Suppose to the contrary that $S \subseteq Q(\omega)$. It can be observed the point that there exists a state $\omega_{0} \in Q(\omega) \backslash S$ such that $Q(\xi) \backslash S=Q\left(\omega_{0}\right) \backslash S$ for every $\xi \in Q\left(\omega_{0}\right) \backslash S$ : For if not then, noting that $Q$ satisfies the properties Ref and $\operatorname{Trn}$, we can plainly obtain an infinite sequence $\left\{\omega_{n} \mid n=0,1,2, \cdots\right\}$ of states in $Q(\omega)$ such that $\omega_{n+2}$ belongs to the set $Q\left(\omega_{n+1}\right) \backslash S \subseteq Q\left(\omega_{n}\right) \backslash S \subseteq$ $Q(\omega) \backslash S$ for every $n=0,1,2, \ldots$, in contradiction to the assumption that $\Omega$ is finite as required. Therefore, we have verified that $A\left(\omega_{0}\right)=Q\left(\omega_{0}\right) \backslash S$.

In viewing that $\omega_{0} \in Q(\omega) \subseteq\{\xi \in \Omega \mid f(Q(\xi))=f(Q(\omega))\}$, we conclude that $A\left(\omega_{0}\right) \in \mathcal{S}$ in final contradiction.

Case II. $\mathcal{S}=\emptyset$ : By the similar argument as above, it can be verified that there exists a state $\omega_{0} \in Q(\omega)$ such that $A\left(\omega_{0}\right)=Q\left(\omega_{0}\right) \subseteq\{\xi \in$ $\Omega \mid f(Q(\xi))=f(Q(\omega))\}$, and hence $A\left(\omega_{0}\right) \in \mathcal{S}$ in contradiction. This establishes the proof of Fundamental lemma.

\section{Appendix B}

\section{Proof of Proposition 1}

Set $[q] \cap B_{C}([q])$ by $H$. This is public belief because $[q] \cap B_{C}([q]) \subseteq B_{E}([q] \cap$ $\left.B_{C}([q])\right)$ by Axiom FP, and so $H$ is $P_{i}$-invariant for every $i$. We can plainly observe that $H$ satisfies the conditions (a) and (b) in Fundamental lemma. Therefore $\mu(X \mid H)=\mu\left(X \cap A_{i}(X) \mid H\right)=q_{i}$ for every $i$.

\section{Notes}

1. The axiom PL is due to E. Dekel, B. L. Lipman and A. Rustichini [3]. 
2. The strong epistemic model is a tuple $\left\langle\Omega,\left(K_{i}\right)_{i \in N}\right\rangle$, in which $\Omega$ is a state-space and $K_{i}$ is $i$ 's knowledge operator satisfying the following axioms: For every $E, F$ of $\mathcal{F}$,
$\mathbf{N} \quad K_{i} \Omega=\Omega$
$\mathbf{K} \quad K_{i}(E \cap F)=K_{i} E \cap K_{i} F ;$
T $\quad K_{i} F \subseteq F ;$
$4 \quad K_{i} F \subseteq K_{i} K_{i} F$;
$5 \quad \Omega \backslash K_{i} F \subseteq K_{i}\left(\Omega \backslash K_{i} F\right)$.

3. $i$ 's information partition $\Pi_{i}$ induced from the knowledge operator $K_{i}$ is defined by $\Pi_{i}(\omega)=\bigcap_{T \in \mathcal{F}}\left\{T \in \mathcal{F} \mid \omega \in K_{i} T\right\}$

4. See Section 4 in Matsuhisa and Usami [9] for the discussion why the improvement of the notion of posterior is needed.

5. C.f. Bacharach $[2]$

6. That is, there exists a natural number $m$ such that for all $t, s(t)=s(t+m)$

7. Where a natural number $k(k=1,2, \ldots, 8)$ denotes the state $\omega_{k}$ for simplicity.

8. The $R T$-information structure stands for reflexive and transitive information structure

9. A similar lemma may be found in Matsuhisa and Kamiyama [7] and Matsuhisa and Usami [9].

\section{References}

1. Aumann,R.J. Agreeing to disagree. Annals of Statistics, 4:1236-1239, 1976.

2. Bacharach,M. Some extensions of a claim of Aumann in an axiomatic model of knowledge. Journal of Economic Theory, 37:167-190, 1985.

3. Dekel,E., Lipman,B.L. and Rustichini,A. Standard state-space models preclude unawareness. Econometrica, 66:159-173, 1998

4. Fagin,R., Halpern,J.Y., Moses,Y. and Vardi, M.Y. Reasoning about Knowledge. The MIT Press, Cambridge, Massachusetts, London, England, 1995.

5. Geanakoplos,J.D. and Polemarchakis,H.M. We can't disagree forever. Journal of Economic Theory 28:192-200, 1982.

6. Krasucki,P. Protocol forcing consensus. Journal of Economic Theory, 70:266-272, 1996.

7. Matsuhisa,T. and Kamiyama,K. Lattice structure of knowledge and agreeing to disagree. Journal of Mathematical Economics, 27:389-410, 1997.

8. Matsuhisa,T., Tominaga,M. and Kamiyama,K. Communication, consensus and lattice structure of knowledge. Far East Journal of Applied Mathematics, 4(3):239$252,2000$.

9. Matsuhisa,T. and Usami,S.-S. Awareness, belief and agreeing to disagree. Far East Journal of Mathematical Sciences, 2(6):833-844, 2000. 


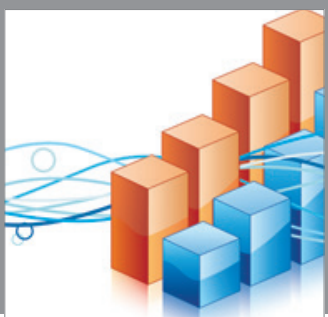

Advances in

Operations Research

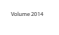

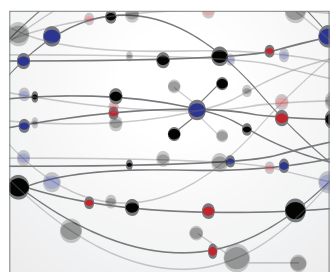

\section{The Scientific} World Journal
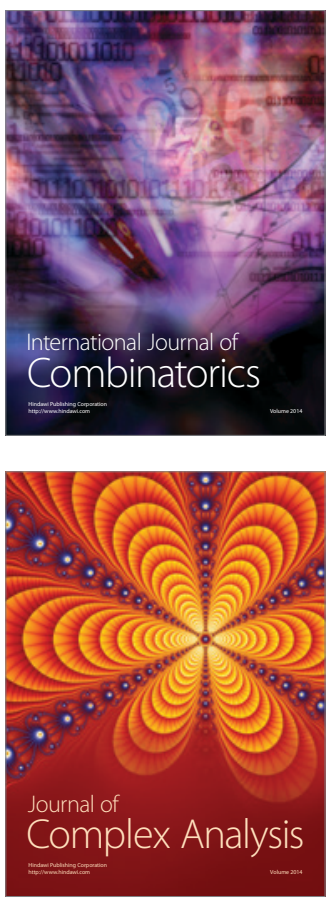

International Journal of

Mathematics and

Mathematical

Sciences
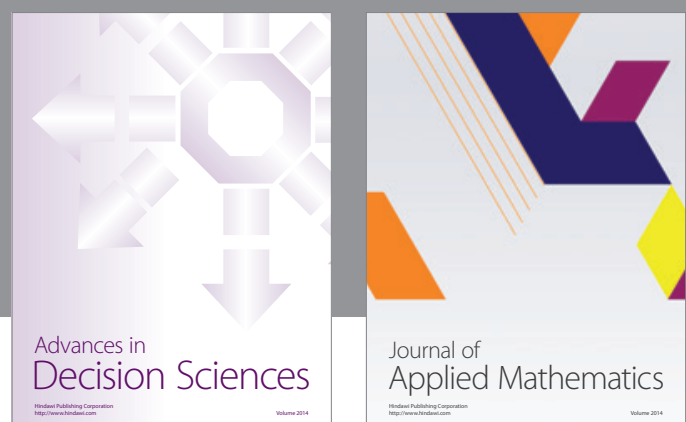

Journal of

Applied Mathematics
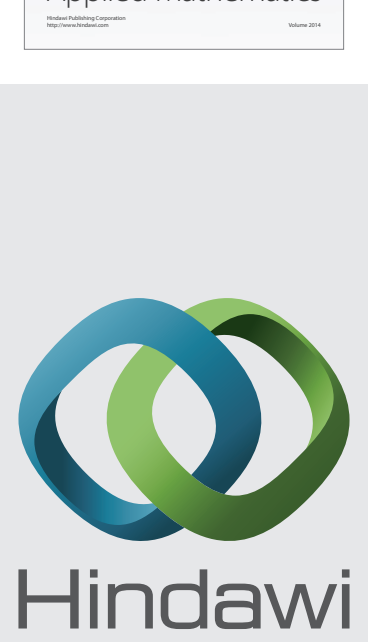

Submit your manuscripts at http://www.hindawi.com
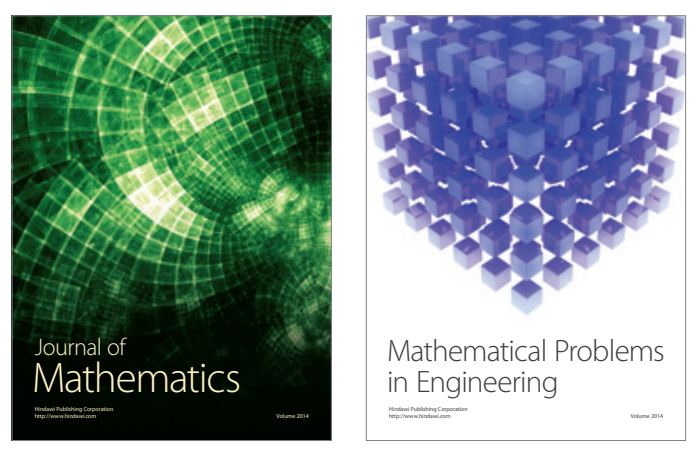

Mathematical Problems in Engineering
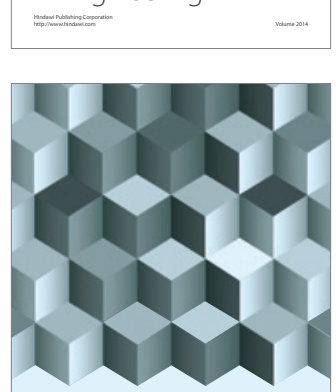

Journal of

Function Spaces
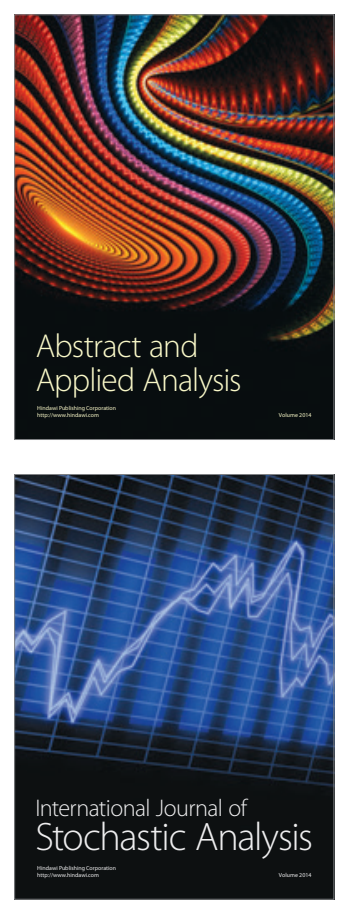

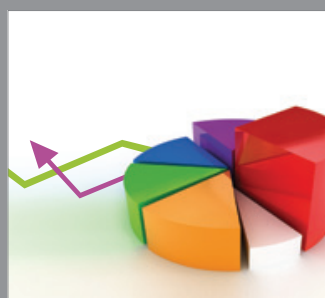

ournal of

Probability and Statistics

Promensencen
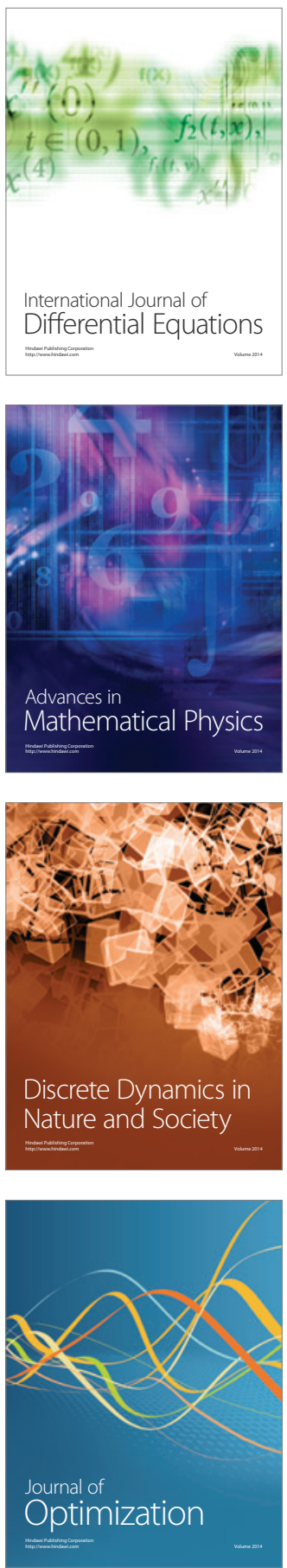